\title{
POLYNOMIAL INTERPOLATION PROBLEM FOR SKEW POLYNOMIALS
}

\begin{abstract}
Aleksandra Lj. Erić
Let $R=K[x ; \sigma]$ be a skew polynomial ring over a division ring $K$. We introduce the notion of derivatives of skew polynomial at scalars. An analogous definition of derivatives of commutative polynomials from $K[x]$ as a function of $K[x] \rightarrow K[x]$ is not possible in a non-commutative case. This is the reason why we have to define the derivative of a skew polynomial at a scalar. Our definition is based on properties of skew polynomial rings, and it makes possible some useful theorems about them. The main result of this paper is a generalization of polynomial interpolation problem for skew polynomials. We present conditions under which there exists a unique polynomial of a degree less then $n$ which takes prescribed values at given points $x_{i} \in K(1 \leq n)$. We also discuss some kind of SILVESTER-LAGRANGE skew polynomial.
\end{abstract}

\section{INTRODUCTION}

Let $K$ be a division ring, and let $\sigma$ be a monomorphism of $K$. For an indeterminate $x$ over $K$, we write $K[x ; \sigma]$ for the ring of skew polynomials over $K$. By this, we mean that $K[x ; \sigma]$ is the set of all left polynomials $\sum_{i} c_{i} x^{i}$ which are added in the usual way, and multiplied by using the distributive law together with the rule that $x c=c^{\sigma} x$ for any $c \in K$ ( by $c^{\sigma}$ we will denote $\sigma$-image of $c$ ). Thus, the coefficients need not commute with the variable $x$. The fact that $(a b)^{\sigma}=a^{\sigma} b^{\sigma}$ guarantees the associative law for polynomial multiplications, so $K[x ; \sigma]$ is a ring. This so-called skew polynomial ring is a basic object of study in noncommutative ring theory $[\mathbf{1}]$. As it is easily seen,the usual division algorithm stays in $R=K[x ; \sigma]$ : For $f(x) \in R$ and $g(x) \in R \backslash\{0\}$, we can uniquely define $f(x)=h(x) g(x)+r(x)$, where $r(x)=0$

2000 Mathematics Subject Classification. 16S36,16U30,15A03.

Key Words and Phrases. Interpolation, skew polynomials. 
or $\operatorname{deg} r(x)<\operatorname{deg} g(x)$. In particular, $R$ is a left PID (principal ideal domain) i.e. any nonzero left ideal $I$ has the form $R g$; here, $g$ is any polynomial in $I$ of the smallest degree [2].

To define evaluation of the left polynomials at scalar, it suffices to recall some of its main properties as follows.

- The remainder Theorem [3]: $f(x)=q(x)(x-a)+f(a)$ where $q(x)$ is uniquely determined by $f$ and by $a$. From this it follows that $f$ is divisible by $x-a$ iff $f(a)=0$. In this case, we say that $a$ is right root of $f$.

- The Product Formula [3] for evaluating $f=g h$ at any $d \in K$ :

$$
f(d)=\left\{\begin{array}{cl}
0 & \text { if } h(d)=0 \\
g\left(d^{h(d)}\right) h(d) & \text { if } h(d) \neq 0
\end{array}\right.
$$

Here, $a^{c}$ is the $\sigma$-conjugate of $a$ by $c$, and it is defined by $a^{c}=\sigma(c) a c^{-1}$, for any $c \in K^{*}$.

- The evaluating formula [3]: if $f(x)=\sum_{i} a_{i} x^{i}$, then $f(a)=\sum_{i} a_{i} N_{i}(a)$ for all $a \in K$ where $N_{0}(a)=1$, and $N_{n}(a)=\sigma^{n-1}(a) \ldots \sigma(a) a$.

\section{EVALUATING DERIVATIVES OF SKEW POLYNOMIALS AT} SCALAR

Let $f(x) \in R=K[x ; \sigma]$. By dividing $f(x)$ by polynomial $(x-d)^{2}=x^{2}-$ $\left(d+d^{\sigma}\right) x+d^{2}$ we get the remainder $c x+r$.

We can define the first derivative of polynomial $f(x)$ at scalar $d: f^{\prime}(d)$ to be $c$. We will denote by $M_{n}(d)$ the first derivative of polynomial $x^{n}$ at scalar $d$. For example:

$$
x^{2}=(x-d)^{2}+\left(d+d^{\sigma}\right) x-d^{2} .
$$

So, $M_{2}(d)=d+d^{\sigma}$. Also from

$$
x^{3}=\left(x+d^{\sigma}+d^{\sigma^{2}}\right)(x-d)^{2}+\left(d^{\sigma^{2}} d^{\sigma}+d^{\sigma^{2}} d+d^{\sigma} d\right) x-\left(d^{\sigma}+d^{\sigma^{2}}\right) d^{2}
$$

we get

$$
M_{3}(d)=d^{\sigma^{2}} d^{\sigma}+d^{\sigma^{2}} d+d^{\sigma} d
$$

Definition 1. Let $f(x)=\sum_{i} c_{i} x^{i} \in R$. Then $f^{\prime}(d)=\sum_{i} c_{i} M_{i}(d)$, where $M_{0}(d)=0$, $M_{1}(d)=1$ and

$$
M_{i}(d)=\sum_{i-1 \geq k_{1}>k_{2}>\cdots>k_{i-1} \geq 0} d^{\sigma^{k_{1}}} \cdots d^{\sigma^{k_{i-1}}}
$$


Note that, if $\sigma=1$, then $M_{i}(d)=i d^{i-1}$. So $f^{\prime}(d)$ is the usual evaluation of derivative of $f$.

ExAmple. Let $R=\mathbb{C}\left[x ;^{-}\right]$. Then

$$
M_{2}(d)=d+\bar{d}, \quad M_{3}(d)=d^{2}+2|d|^{2}, \quad M_{4}(d)=2|d|^{2}(d+\bar{d}) .
$$

If $f(x)=i x^{2}+(2+i) x-3$, then $f^{\prime}(2-i)=2+5 i$.

If $f(x)=x^{3}-(1+i) x^{2}-x+1+i=(x-1-i)(x-i)^{2}$, then, $f^{\prime}(i)=0, f(i)=0$.

If $f(x)=x^{4}-20 x$, then $f^{\prime}(1+2 i)=0$ and $f(1+2 i)=5-40 i$.

ExAmple. Let $R=\mathbb{R}(t)[x ; \sigma], \sigma: f(t) \mapsto f\left(t^{2}\right)$. Then

$$
M_{2}(f(t))=f(t)+f\left(t^{2}\right), \quad M_{3}(f(t))=f\left(t^{4}\right) f\left(t^{2}\right)+f\left(t^{4}\right) f(t)+f\left(t^{2}\right) f(t) .
$$

If $p(x)=(x+t)(x-t)^{2}=x^{3}+\left(t-t^{2}-t^{4}\right) x^{2}+\left(t^{4}-t^{3}-t^{2}\right) x+t^{3}$, then

$$
\begin{aligned}
p(t) & =t^{7}+\left(t-t^{2}-t^{4}\right) t^{3}+\left(t^{4}-t^{3}-t^{2}\right) t+t^{3}=0, \\
p^{\prime}(t) & =\left(t^{6}+t^{5}+t^{3}\right)+\left(t-t^{2}-t^{4}\right)\left(t+t^{2}\right)+\left(t^{4}-t^{3}-t^{2}\right)=0 .
\end{aligned}
$$

Proposition 2.1. Let $f(x), g(x) \in R=K[x ; \sigma]$ and $d \in K$. Then

$$
(f+g)^{\prime}(d)=f^{\prime}(d)+g^{\prime}(d) .
$$

Proof. For $f(x)=\sum_{i} a_{i} x^{i}$ and $g(x)=\sum_{i} b_{i} x^{i}$ (we can assume that the polynomials are of the same degree) $(f+g)(x)=\sum_{i}\left(a_{i}+b_{i}\right) x^{i}$, so

$$
(f+g)^{\prime}(d)=\sum_{i}\left(a_{i}+b_{i}\right) M_{i}(d)=\sum_{i} a_{i} M_{i}(d)+\sum_{i} b_{i} M_{i}(d)=f^{\prime}(d)+g^{\prime}(d) .
$$

Proposition 2.2. $M_{i+j}(d)=N_{j}(d)^{\sigma^{i}} M_{i}(d)+M_{j}(d)^{\sigma^{i}} N_{i}(d)$.

Proof. $M_{i+1}(d)=d^{\sigma^{i}} M_{i}(d)+N_{i}(d)$ because of

$$
\begin{aligned}
M_{i+1}(d) & =\sum_{i \geq k_{1}>k_{2}>\cdots>k_{i} \geq 0} d^{\sigma^{k_{1}}} \cdots d^{\sigma^{k_{i}}} \\
& =d^{\sigma^{i}} \sum_{i-1 \geq k_{2}>k_{3}>\cdots>k_{i} \geq 0} d^{\sigma^{k_{2}}} \cdots d^{\sigma^{k_{i}}}+\sum_{i-1 \geq k_{1}>k_{2}>\cdots>k_{i} \geq 0} d^{\sigma^{k_{1}}} \cdots d^{\sigma^{k_{i}}} \\
& =d^{\sigma^{i}} M_{i}(d)+d^{\sigma^{i-1}} \ldots d=d^{\sigma^{i}} M_{i}(d)+N_{i}(d) .
\end{aligned}
$$

We proceed by induction on $j$. The case $j=1$ follows from the first expression. Now, suppose that the proposition is true for some $j$, i. e.

$$
M_{i+j+1}(d)=d^{\sigma^{i+j}} M_{i+j}(d)+N_{i+j}(d) .
$$

Then

$$
M_{i+j+1}(d)=d^{\sigma^{i+j}} N_{j}(d)^{\sigma^{i}} M_{i}(d)+d^{\sigma^{i+j}} M_{j}(d)^{\sigma^{i}} N_{i}(d)+N_{i+j}(d)
$$


Applying

$$
\begin{aligned}
\left(d^{\sigma^{j}} N_{j}(d)\right)^{\sigma^{i}} & =\left(N_{j+1}(d)\right)^{\sigma^{i}} \\
N_{i+j}(d) & =N_{j}(d)^{\sigma^{i}} N_{i}(d), \\
d^{\sigma^{i+j}} M_{j}(d)^{\sigma^{i}}+N_{j}(d)^{\sigma^{i}} & =M_{j+1}(d)^{\sigma^{i}}
\end{aligned}
$$

we get

$$
M_{i+j+1}(d)=N_{j+1}(d)^{\sigma^{i}} M_{i}(d)+M_{j+1}(d)^{\sigma^{i}} N_{i}(d)
$$

Proposition 2.3. Let $f(x)=g(x) h(x) \in R=K[x ; \sigma], g(x)=\sum_{i} b_{i} x^{i}$ and $h(x)=$ $\sum_{j} c_{j} x^{j}$. Then

$$
f^{\prime}(d)=\sum_{i} b_{i} a^{\sigma^{i}} M_{i}(d)+g\left(e^{\sigma} d e^{-1}\right) e,
$$

where $a=h(d), e=h^{\prime}(d)$ and $e \neq 0$.

Proof. Since $f(x)=\sum_{i, j} b_{i} c_{j}^{\sigma^{i}} x^{i+j}$, we have

$$
\begin{aligned}
f^{\prime}(d) & =\sum b_{i} c_{j}^{\sigma^{i}} M_{i+j}(d) \\
& =\sum_{i, j} b_{i} c_{j}^{\sigma^{i}} N_{j}(d)^{\sigma^{i}} M_{i}(d)+\sum_{i, j} b_{i} c_{j}^{\sigma_{i}} M_{j}\left(d^{\sigma^{i}}\right) N_{i}(d) \\
& =\sum_{i, j} b_{i}\left(c_{j} N_{j}(d)\right)^{\sigma^{i}} M_{i}(d)+\sum_{i, j} b_{i}\left(c_{j} M_{j}(d)\right)^{\sigma^{i}} N_{i}(d) \\
& =\sum_{i} b_{i} a^{\sigma^{i}} M_{i}(d)+\sum_{i} b_{i} e^{\sigma^{i}} N_{i}(d) .
\end{aligned}
$$

Therefore,

$$
\sum_{i, j} b_{i} e^{\sigma^{i}} N_{i}(d)=\sum_{i, j} b_{i} N_{i}\left(e^{\sigma} d e^{-1}\right) e=g\left(e^{\sigma} d e^{-1}\right) e
$$

If $\sigma=1$, then $f^{\prime}(d)=g^{\prime}(d) h(d)+g(d) h^{\prime}(d)$, which is the usual formula for a derivative of product.

Theorem 2.4. Let $f(x) \in R=K[x ; \sigma]$ and $d \in K$. Then

$$
f(x)=g(x)(x-d)^{2}
$$

for some $g(x) \in R=K[x ; \sigma]$ iff $f(d)=f^{\prime}(d)=0$.

The proof is easy and thus omitted. 


\section{EVALUATING DERIVATIVES OF THE HIGHER ORDER OF SKEW POLYNOMIALS AT SCALAR}

The derivative of order $n$ of polynomial $x^{i}$ at $d \in K$ for $1<i<n$ is $M_{i}^{n}(d)=n ! \sum_{i-1 \geq k_{1}>k_{2}>\cdots>k_{i-n} \geq 0} d^{\sigma^{k_{1}}} \cdots d^{\sigma^{k_{i-n}}}, M_{n}^{n}(d)=n !$ and $M_{i}^{n}(d)=0$ for $n>i$.

We get it as $n ! A_{n}$ where $A_{n}$ is from

$$
x^{i}=q(x)(x-d)^{n+1}+A_{n} x^{n}+\ldots+A_{0} .
$$

For example,

$$
\begin{aligned}
& (x-d)^{3}=x^{3}-\left(d^{\sigma^{2}}+d^{\sigma}+d\right) x^{2}+\left(\left(d^{\sigma}\right)^{2}+d^{2}+d d^{\sigma}\right) x-d^{3}, \\
& x^{3}=(x-d)^{3}+\left(d^{\sigma^{2}}+d^{\sigma}+d\right) x^{2}-\left(\left(d^{\sigma}\right)^{2}+d^{2}+d d^{\sigma}\right) x+d^{3} .
\end{aligned}
$$

and so, $M_{3}^{2}(d)=2\left(d^{\sigma^{2}}+d^{\sigma}+d\right)$.

Example. Let $R=\mathbb{C}\left[x ;^{-}\right]$and $f(x)=x^{3}+(1+i) x^{2}-x-(1+i)$ from $R$. Then $M_{3}^{2}(d)=2(d+\bar{d}+d)=2(2 d+\bar{d})$. Here we have $f(1+i)=0, f^{\prime}(1+i)=5+3 i$, $f^{\prime \prime}(1+i)=8+4 i$. For $f(x)=x^{3}-i x^{2}-x+i=(x-i)^{3}$ we have $f(i)=f^{\prime}(i)=f^{\prime \prime}(i)=0$.

Definition 2. The $n$-th derivative of polynomial $f(x) \in R=K[x ; \sigma], f(x)=$ $\sum_{i} c_{i} x^{i}$ at $d \in K$ is

$$
f^{(n)}(d)=\sum_{i} c_{i} M_{i}^{n}(d)
$$

Note that $M_{i}^{n}(d)=0$ if $n>i$.

Proposition 3.1. For $d \in K, n>1$, we have

(1) $M_{i+1}^{n}(d)=d^{\sigma^{i}} M_{i}^{n}(d)+n M_{i}^{n-1}(d)$.

(2) $M_{i+j}^{n}(d)=\sum_{k=0}^{n}\left(\begin{array}{l}n \\ k\end{array}\right) M_{j}^{k}\left(d^{\sigma^{i}}\right) M_{i}^{n-k}(d)$.

Proof. (1)

$$
\begin{aligned}
M_{i+1}^{n}(d) & =n ! \sum_{i \geq k_{1}>k_{2}>\cdots>k_{i-n+1} \geq 0} d^{\sigma^{k_{1}}} \cdots d^{\sigma^{k_{i-n+1}}} \\
& =n !\left(d^{\sigma^{i}} \frac{M_{i}^{n}(d)}{n !}+\frac{M_{i}^{n-1}(d)}{(n-1) !}\right)=d^{\sigma^{i}} M_{i}^{n}(d)+n M_{i}^{n-1}(d) .
\end{aligned}
$$

(2) We proceed by induction. In case $j=1$ it is (1).

$$
\begin{aligned}
& M_{i+j+1}^{n}(d)= d^{\sigma^{i+j}} M_{i+j}^{n}(d)+ \\
&=\sum_{k=0}^{n}\left(\begin{array}{l}
n \\
k
\end{array}\right) d^{\sigma^{i+j}} M_{j}^{k}\left(d^{\sigma^{i}}\right) M_{i}^{n-k}(d) \\
& \quad+n \sum_{k=0}^{n-1}\left(\begin{array}{c}
n-1 \\
k
\end{array}\right) M_{j}^{k}\left(d^{\sigma^{i}}\right) M_{i}^{n-k-1}(d) .
\end{aligned}
$$


From $(1), M_{j+1}^{k}\left(d^{\sigma^{i}}\right)=d^{\sigma^{i+j}} M_{j}^{k}\left(d^{\sigma^{i}}\right)+k M_{j}^{k-1}\left(d^{\sigma^{i}}\right)$, so

$$
\begin{aligned}
M_{i+j+1}^{n}(d)=\sum_{k=0}^{n}\left(\begin{array}{l}
n \\
k
\end{array}\right) M_{j+1}^{k}\left(d^{\sigma^{i}}\right) M_{i}^{n-k}(d)-\sum_{k=0}^{n}\left(\begin{array}{l}
n \\
k
\end{array}\right) k M_{j}^{k-1}\left(d^{\sigma^{i}}\right) M_{i}^{n-k}(d) & \\
& +n \sum_{k=0}^{n-1}\left(\begin{array}{c}
n-1 \\
k
\end{array}\right) M_{j}^{k}\left(d^{\sigma^{i}}\right) M_{i}^{n-k-1}(d) \\
n \sum_{k=0}^{n-1}\left(\begin{array}{c}
n-1 \\
k
\end{array}\right) M_{j}^{k}\left(d^{\sigma^{i}}\right) M_{i}^{n-k-1}(d)= & n \sum_{k=1}^{n}\left(\begin{array}{l}
n-1 \\
k-1
\end{array}\right) M_{j}^{k-1}\left(d^{\sigma^{i}}\right) M_{i}^{n-k}(d) \\
= & \sum_{k=1}^{n}\left(\begin{array}{l}
n \\
k
\end{array}\right) k M_{j}^{k-1}\left(d^{\sigma^{i}}\right) M_{i}^{n-k}(d)
\end{aligned}
$$

So, $M_{i+j+1}^{n}(d)=\sum_{k=0}^{n}\left(\begin{array}{l}n \\ k\end{array}\right) M_{j+1}^{k}\left(d^{\sigma^{i}}\right) M_{i}^{n-k}(d)$. If $f(x)=g(x) h(x)=\sum_{i, j} b_{i} c_{j}^{\sigma^{i}} x^{i+j}$, then

$$
\begin{aligned}
f^{(n)}(d) & =\sum_{i, j} b_{i} c_{j}^{\sigma^{i}} M_{i+j}^{n}(d)=\sum_{i, j, k}\left(\begin{array}{l}
n \\
k
\end{array}\right) b_{i} c_{j}^{\sigma^{i}} M_{j}^{k}\left(d^{\sigma^{i}}\right) M_{i}^{n-k}(d) \\
& =\sum_{i, j, k}\left(\begin{array}{l}
n \\
k
\end{array}\right) b_{i}\left(c_{j} M_{j}^{k}(d)\right)^{\sigma^{i}} M_{i}^{n-k}(d)=\sum_{i, k} n k b_{i} a_{k}^{\sigma^{i}} M_{i}^{n-k}(d),
\end{aligned}
$$

where $a_{k}=g^{(k)}(d)$.

Proposition 3.2. Let $g(x)=(x-d)^{n}$. Then $g(d)=\cdots=g^{(n-1)}(d)=0$.

Proof. We prove the proposition by induction. In case $n=1$ it is easy verified. Suppose that Proposition is true for any $k<n$. Let $g(x)=(x-d)^{n+1}=p(x) q(x)$, where $p(x)=(x-d)$ and $q(x)=(x-d)^{n}$. Then, for $0 \leq \ell \leq n-1$

$$
g^{(\ell)}(d)=\sum\left(\begin{array}{l}
\ell \\
k
\end{array}\right) b_{i} a_{k}^{\sigma^{i}} M_{i}^{\ell-k}(d)
$$

where $a_{k}=q^{k}(d)$, so $a_{k}=0$ for $0 \leq k \leq n-1$.

Then $g^{(i)}(d)=0$ for $0 \leq i \leq n-1$. We still need to prove $g^{(n)}(d)=0$.

$$
\begin{aligned}
g(x) & =(x-d)^{n+1}=x^{n+1}-\left(d+d^{\sigma}+\cdots+d^{\sigma^{n}}\right) x^{n}+\cdots, \\
g^{(n)}(d) & =M_{n+1}^{n}-\left(d+d^{\sigma}+\cdots+d^{\sigma^{n}}\right) M_{n}^{n}(d), \\
M_{n}^{n}(d) & =n ! \\
M_{n+1}^{n}(d) & =n !\left(d+d^{\sigma}+\cdots+d^{\sigma^{n}}\right) .
\end{aligned}
$$

So, $g^{n}(d)=0$.

Theorem 3.3. Let $f(x) \in K[x ; \sigma]$ and $d \in K$. Then

$$
f(x)=g(x)(x-d)^{n} \text { for some } g(x)
$$


iff $f(d)=f^{\prime}(d)=\cdots=f^{n-1}(d)=0$.

Proof. Assume $f(d)=f^{\prime}(d)=\cdots=f^{n-1}(d)=0$.Then

$$
f(x)=g(x)(x-d)^{n}+a_{0}+a_{1} x+\cdots+a_{n-1} x^{n-1} \quad\left(a_{i} \in K\right)
$$

For $G(x)=g(x)(x-d)^{n}$ we have

$$
G^{k}(d)=\sum_{i} b_{i} a_{k}^{\sigma^{i}} M_{i}^{n-1}(d),
$$

where $b_{i}$ are coefficients of polynomial $g(x)$ and $a_{k}$ is the $k$-th derivative of polynomial $(x-d)^{n}$. So $a_{k}=0$, then $G^{k}(d)=0 \quad 0 \leq k \leq n-1$

$$
\begin{aligned}
& 0=f(d)=a_{n-1} N_{n-1}(d)+\ldots+a_{1} N_{1}(d)+a_{0}, \\
& 0=f^{\prime}(d)=a_{n-1} M_{n-1}(d)+\ldots+a_{1} M_{1}(d), \\
& 0=f^{\prime \prime}(d)=a_{n-1} M_{n-1}^{2}(d)+\ldots+a_{2} M_{2}^{2}(d), \\
& \quad \vdots \\
& 0=f^{n}(d)=a_{n-1} M_{n-1}^{n-1}(d),
\end{aligned}
$$

$M_{n}^{n}(d)=(n-1)$ ! implies $a_{n-1}=0$ and by solving the system, we get $a_{i}=0$ for all $i$ i.e. $f(x)=g(x)(x-d)^{n}$. The converse is easy to verify from Proposition 3.2 and properties of derivatives.

Theorem 3.4. Let $f(x) \in K[x ; \sigma]$ and $\operatorname{deg} f=n$. Then

$(*) \quad f(x)=f(d)+\frac{f^{\prime}(d)}{1 !}(x-d)+\frac{f^{\prime \prime}(d)}{2 !}(x-d)^{2}+\cdots+\frac{f^{(n)}(d)}{n !}(x-d)^{n}$.

Proof. We proceed by induction on the degree of $f$. Let $\operatorname{deg} f=1$. Then $f(x)=A(x-d)+f(d)$ and $f^{\prime}(d)=A$, so

$$
f(x)=f(d)+\frac{f^{\prime}(d)}{1 !}(x-d) .
$$

Assume that $(*)$ holds, for any polynomial $f$ with $\operatorname{deg} f=n$.

Let $f(x)$ be a polynomial with $\operatorname{deg} f=n+1$. Then $f(x)=g(x)(x-d)+f(d)$ for some $g(x)$ and $\operatorname{deg} g=n$. Then

$$
f^{(m)}(d)=(g(x)(x-d))^{(m)}(d) .
$$

Using the product formula, we obtain

$$
(g(x)(x-d))^{(m+1)}(d)=\sum_{i, k}\left(\begin{array}{c}
m+1 \\
k
\end{array}\right) b_{i} a_{k}^{\sigma^{i}} M_{i}^{m+1-k}(d),
$$

where $b_{i}$ are coefficients of $g$ and $a_{k}$ value of $k$-th derivative of polynomial $(x-d)$ at $d$. So $a_{1}=1$ and $a_{i}=0$ for $i>1$, which implies: $(g(x)(x-d))^{(m+1)}(d)=$ 
$(m+1) \sum_{i} b_{i} M_{i}^{m}(d)=(m+1) g^{(m)}(d)$. So, $(m+1) g^{(m+1)}(d)=f^{(m+1)}(d)$ and finally, we get $(*)$ for $n$ replace with $n+1$.

\section{POLYNOMIAL INTERPOLATION FOR SKEW POLYNOMIALS}

For a field $K$, it is well known that for $x_{0}, \ldots, x_{n-1}, x_{n}$ being different elements of $K$ and $y_{0}, \ldots, y_{n-1}, y_{n} \in K$, there exists the unique polynomial $f \in K[x]$ such that $f\left(x_{i}\right)=y_{i}$ and $\operatorname{deg} f \leq n$. However, the condition $x_{i} \neq x_{j}$ is not sufficient for existence of such a polynomial in a non-commutative case.

Let us first mention some facts about skew polynomials.

Proposition 4.1. Let $\Delta=\left\{x_{0}, \ldots, x_{n}\right\}$ and $x_{i} \in K$, where $K$ is a division ring. Then

(1) There exists the nonzero polynomial $f \in K[x ; \sigma]$ such that $f\left(x_{i}\right)=0$.

(2) The set I of polynomials vanishing on $\Delta$ form a left ideal in $K[x ; \sigma]$.

(3) If $f_{\Delta}$ is monic polynomial of the smallest degree in $I$, then $I=R f_{\Delta}$, where $R=K[x ; \sigma]$. We will call $f_{\Delta}$ minimal polynomial of $\Delta$.

Proof. (1) Let $\Delta$ be doubleton i.e. $\Delta=\{a, b\}$. Then, a polynomial $f$ vanishing on $\Delta$ is

$$
f(x)=\left(x-\sigma(b-a) b(b-a)^{-1}\right)(x-a),
$$

which follows from Product and Remainder Theorem.

If $g$ is a polynomial vanishing on $\Gamma=\left\{x_{0}, \ldots, x_{n-1}\right\}$, then a polynomial $f$ vanishing on $\Delta=\Gamma \cup\left\{x_{n}\right\}$ is

$$
f(x)=\left(x-\sigma\left(g\left(x_{n}\right)\right) x_{n} g\left(x_{n}\right)^{-1}\right) g(x) .
$$

(2) If $f, g \in I$, then $f(d)=g(d)=0$ for all $d \in \Delta$. So, $(f+g)(d)=$ $f(d)+g(d)=0$. Also, for $\alpha \in R(\alpha f)(d)=\alpha\left(\sigma(f(d)) d f(d)^{-1}\right) f(d)=0$ (from Product formula). Then $I$ is the left ideal in left PID, so it is principal.

(3) If $f \in I$, then $f\left(x_{i}\right)=0$ for all $x_{i} \in \Delta$. $f=q f_{\Delta}+r$ where $f_{\Delta}$ is a polynomial of minimal degree in $I, q, r \in K[x ; \sigma]$ and $\operatorname{deg} r<\operatorname{deg} f_{\Delta}$. From $f\left(x_{i}\right)=r\left(x_{i}\right)=0$ it follows $r \equiv 0$. The conclusion is that $f \in R f_{\Delta}$.

Theorem 4.2. Let $\Delta=\left\{x_{0}, \ldots, x_{n}\right\}$ and $x_{i} \in K$ where $K$ is a division ring. For any $y_{0}, \ldots, y_{n} \in K$ there exists a unique polynomial $f \in R$ such that $f\left(x_{i}\right)=y_{i}$ and $\operatorname{deg} f \leq n$ if and only if $\operatorname{deg} f_{\Delta}=n+1$ where $f_{\Delta}$ is the minimal polynomial of the set $\Delta$.

Proof. Let $\Phi: R=K[x ; \sigma] \rightarrow K^{n+1}$ be a $K$-linear function of the left $K$-spaces such that

$$
f \mapsto\left(f\left(x_{0}\right), \ldots, f\left(x_{n}\right)\right) .
$$

The kernel of the homomorphism $\Phi$ consists of all polynomials $f$ such that $f\left(x_{i}\right)=0$ for all $i$. So, $\operatorname{Ker} \Phi=R f_{\Delta}$, where $f_{\Delta}$ is the minimal polynomial of the set $\Delta$. Then 
$\operatorname{Im} \Phi \cong R / \operatorname{Ker} \Phi([4]$, Th. 2.1).

$$
\operatorname{dim} \operatorname{Im} \Phi=\operatorname{dim} R / \operatorname{Ker} \Phi=\operatorname{dim} R / R f_{\Delta}=\operatorname{deg} f_{\Delta}
$$

$\operatorname{dim} R / R f_{\Delta}=\operatorname{deg} f_{\Delta}$ because for $m=\operatorname{deg} f_{\Delta},\left\{1, x, \ldots, x^{m-1}\right\}$ is a base of the left $K$-space $R / R f_{\Delta}$. Indeed, $g=q f_{\Delta}+r$ and $\operatorname{deg} r<m$.

The homomorphism $\Phi$ is surjective iff $\operatorname{dim} \operatorname{Im} \Phi=n+1$, which means that $\operatorname{deg} f_{\Delta}=n+1$. Then, the interpolation polynomial for such a set $\Delta$ is

$$
f(x)=\sum_{i=0}^{n} y_{i} L_{i}\left(x_{i}\right)^{-1} L_{i}(x),
$$

where $L_{i}(x)$ is the monic polynomial such that $L_{i}\left(x_{j}\right)=0$ for $i \neq j$. The degree of the polynomial $\mathrm{f}$ is $\leq n$. If there is another polynomial $g \operatorname{deg} g \leq n$ and $g\left(x_{i}\right)=y_{i}$, then $(f-g)\left(x_{i}\right)=0$ implies $f-g \in R f_{\Delta}$, so deg $\geq n+1$ and this is impossible.

Example. Let $R=\mathbb{C}\left[x ;^{-}\right]$and $\Delta=\{1, i,-1\}$. Then, the minimal polynomial of $\Delta$ is $f_{\Delta}=x^{2}-1$ of degree 2. It means that for this set, Theorem 4.2 does not hold. For example, there is no polynomial $f$ such that $f(1)=1, f(i)=0$ and $f(-1)=-1$. Also, there are many polynomials such that $f(1)=1, f(i)=0$ and $f(-1)=i$.

$$
f(x)=a x^{2}+\frac{1+i}{2} x+\left(\frac{1-i}{2}-a\right) .
$$

ExAmple. Let $R=\mathbb{C}\left[x ;^{-}\right]$and $\Delta=\{1, i, 2 i\}$. Then, the minimal polynomial of $\Delta$ is $f_{\Delta}=x^{3}-2 i x^{2}-x+2 i$. (We get it from $\left(x-\overline{g(2 i)} 2 i(g(2 i))^{-1}\right) g(x)$ where $\left.g(x)=x^{2}-1\right)$. The degree of minimal polynomial is 3 so, there is a unique polynomial $f$ of degree $\leq 2$ such that $f(1)=A, f(2 i)=B$ and $f(-1)=C$ for any $A, B, C$.

$$
\begin{aligned}
& L_{0}(x)=\left(x-\bar{i} 2 i i^{-1}\right)(x-i)=(x+2 i)(x-i)=x^{2}+3 i x+2, \\
& L_{1}(x)=\left(x-\overline{(2 i-1)} 2 i(2 i-1)^{-1}\right)(x-1)=x^{2}+\frac{1}{5}(3+6 i) x-\frac{2}{5}(4+3 i), \\
& L_{2}(x)=\left(x-\overline{(i-1)} i(i-1)^{-1}\right)(x-1)=(x+1)(x-1)=x^{2}-1 .
\end{aligned}
$$

Then,

$f(x)=\left(\frac{1-i}{6} A+\frac{i-3}{6} B+\frac{1}{3} C\right) x^{2}+\left(\frac{1+i}{2} A-\frac{1+i}{2} B\right) x+\left(\frac{1-i}{3} A+\frac{3+i}{3} B-\frac{1}{3} C\right)$.

ExAmple. Let $R=D[x]$ where $D$ is a division field of real quaternions. If $\Delta=\{i, j, k\}$, then $f_{\Delta}=x^{2}+1$ is a polynomial of degree 2 . There is no polynomial of degree $\leq 2$ such that $f(i)=1, f(j)=0$ and $f(k)=0$.

If $\Delta=\{1, i, j\}$, then $f_{\Delta}=(x-1)\left(x^{2}+1\right)=x^{3}-x^{2}+x-1$ is a polynomial of degree 3. Then, there exists a unique polynomial of degree $\leq 3$ such that $f(1)=A, f(i)=B$ and $f(j)=C$.

There is a relation between the interpolation polynomial problem and the 
$\sigma$-VANDERMONDE matrix. We define $\sigma$-VANDERMONDE matrix to be

$$
V_{n}^{\sigma}\left(x_{0}, \ldots, x_{n-1}\right)=\left[\begin{array}{lllc}
1 & 1 & \ldots & 1 \\
N_{1}\left(x_{0}\right) & N_{1}\left(x_{1}\right) & \ldots & N_{1}\left(x_{n-1}\right) \\
N_{2}\left(x_{0}\right) & N_{2}\left(x_{1}\right) & \ldots & N_{2}\left(x_{n-1}\right) \\
\vdots & \vdots & \ddots & \vdots \\
N_{n-1}\left(x_{0}\right) & N_{n-1}\left(x_{1}\right) & \ldots & N_{n-1}\left(x_{n-1}\right)
\end{array}\right] .
$$

T. Y. LAM $[\mathbf{3}]$ proves that the rank of this matrix is same as the degree of a minimal polynomial of the set $\Delta=\left\{x_{0}, \ldots, x_{n-1}\right\}$, and also gives the following useful formulae for computing it:

(1) For any generalized quaternion division algebra $D$ over a field $F$ of characteristics $\neq 2$, then $\operatorname{rank} V=\sum_{i} \min \left\{2,\left|\Delta_{i}\right|\right\}$ where $\Delta=\Delta_{1} \cup \cdots \cup \Delta_{m}$ is the partition of $\Delta$ into $\sigma$-conjugacy classes.

(2) For $\mathbb{C}[x ;-]$ let $\delta_{1}, \ldots, \delta_{m}$ be different values among $|d|, d \in \Delta$. Then rank $\mathrm{V}=\sum_{i=1}^{m} r_{i}$ where $r_{i}=1$ if exactly one element in $\Delta$ has modulus $\delta_{i}$ and $r_{i}=2$ otherwise.

Using this, we can answer the question about a degree of minimal polynomial of given set. Now, we will give an exposition on generalization of polynomial interpolation problem.

Proposition 4.3. Let $\Delta=\left\{x_{1}, \ldots, x_{k}\right\}$ and $S=\left\{\left(r, s_{r}\right) \mid 1 \leq r \leq k, 0 \leq s_{r} \leq n_{r}\right\}$, where $n_{1}, \ldots, n_{k} \in \mathbf{N}$. Then

(1) There exists a nonzero polynomial $f$ such that $f^{\left(s_{r}\right)}\left(x_{r}\right)=0$ for all $\left(r, s_{r}\right) \in S$.

(2) The set I of polynomials such that $f^{\left(s_{r}\right)}\left(x_{r}\right)=0$ for all $\left(r, s_{r}\right) \in S$ form a left ideal in $R$.

(3) If $f_{\Delta, S}$ is a monic polynomial of the smallest degree in $I$, then $I=R f_{\Delta}$. We will call $f_{\Delta, S}$ the minimal polynomial for the pair $(\Delta, S)$.

Proof. (1) For $n_{r}, 1 \leq r \leq k$ the polynomial $f$ has right factor $\left(x-x_{r}\right)^{n_{r}-1}$. It follows from Theorem 3.3. The left factor is determined by the Product Theorem.

(2) If $f, g \in I$, then $f+g \in I$. It follows from additive property of derivatives at scalar. Let $\alpha \in R$, and $f \in I$. Then (from Proposition 3.1.)

$$
(\alpha f)^{\left(s_{r}\right)}\left(x_{r}\right)=\sum_{i, k}\left(\begin{array}{c}
s_{r} \\
k
\end{array}\right) b_{i} a_{k}^{\sigma^{i}} M_{i}^{s_{r}-k}(d),
$$

where $a_{k}=f^{(k)}\left(x_{r}\right)=0$ and $f(x)=\sum b_{i} x^{i}, b_{i} \in K$. So, $(\alpha f)^{\left(s_{r}\right)}\left(x_{r}\right)=0$ for all $s_{r}$ such that $\left(r, s_{r}\right) \in S$. Then $\alpha f \in I$.

(3) Let $f \in I, f=q f_{\Delta, S}+r$, where $q, r \in R$ and $\operatorname{deg} r<\operatorname{deg} f_{\triangle, S}$. Then, $0=f^{\left(s_{r}\right)}\left(x_{r}\right)=r^{\left(s_{r}\right)}\left(x_{r}\right)$ implies $r \in I . f_{\Delta, S}$ is polynomial of the smallest degree in $I$, so $r \equiv 0$. The conclusion is: $f \in R f_{\Delta, S}$. 
Theorem 4.4. Let $\Delta=\left\{x_{1}, \ldots, x_{k}\right\}$ and $S=\left\{\left(r, s_{r}\right) \mid 1 \leq r \leq k, 0 \leq s_{r} \leq n_{r}\right\}$ where $n_{1}, \ldots, n_{k} \in \mathbb{N}$ and $y_{r}^{s_{r}} \in K$. Then, there exists the unique polynomial $f \in R$ of degree $\leq n-1$ where $n=\sum\left(n_{r}+1\right)$, such that $f^{\left(s_{r}\right)}\left(x_{r}\right)=y_{r}^{s_{r}}$ for all pairs $\left(r, s_{r}\right)$, iff the minimal polynomial (in the sense of Proposition 4.3.) $f_{\Delta, S}$ is of degree $n$.

Proof. Let $\Phi: K[x ; \sigma] \rightarrow K^{n}$ be a $K$-linear function of left $K$-spaces given by

$$
f \mapsto\left(f^{\left(s_{r}\right)}\left(x_{r}\right):\left(r, s_{r}\right)\right) \in S .
$$

It follows from properties of derivatives. The rest of the proof is same as the proof of Theorem 4.2.

ExAmple. Let $R=\mathbb{C}[x ;-]$ and $\Delta=\{1, i\}, S=\{(1,0),(2,0),(2,1)\}$. The minimal polynomial of the pair $(\Delta, S)$, i.e. the minimal polynomial such that $f_{\Delta, S}(1)=f_{\Delta, S}(i)=$ $f_{\Delta, S}^{\prime}(i)=0$ is $f_{\Delta, S}(x)=x^{2}-1$. This is polynomial of degree 2 . There is no polynomial $f$ such that $f(1)=1, f(i)=1$ and $f^{\prime}(i)=1$.

Let $\Delta=\{1, i\}, S=\{(1,0),(1,1),(2,0)\}$. The minimal polynomial of the pair $(\Delta, S)$ is $f_{\Delta, S}(x)=\left(x-\overline{(2-2 i)} i(2-2 i)^{-2}\right)(x-1)^{2}=(x+1)(x-1)^{2}=x^{3}+x^{2}-x-1$ $\left(2-2 i\right.$ is value of polynomial $(x-1)^{2}=x^{2}-2 x+1$ at $\left.i\right)$. This polynomial is of degree 3 , so there is a unique polynomial $f$ of degree $\leq 2$ such that $f(1)=A, f(i)=B$ and $f^{\prime}(1)=C$.

$L_{0}(x)$ is the monic polynomial such that $L_{0}(i)=L_{0}^{\prime}(1)=0$, then $L_{0}(x)=x^{2}$ $-2 x-1+2 i . \quad L_{1}(x)$ is the monic polynomial such that $L_{1}(1)=L_{1}(i)=0$. Then $L_{1}(x)=x^{2}-1 . L_{2}(x)$ is the monic polynomial such that $L_{2}(1)=L_{2}^{\prime}(1)=0$. Then $L_{2}(x)=(x-1)^{2}$. We find that

$$
f(x)=\left(-\frac{1+i}{4} A+\frac{B}{2}+\frac{1+i}{4} C\right) x^{2}+\frac{1+i}{2}(A-C) x+\left(\frac{3-i}{4} A-\frac{B}{2}+\frac{1+i}{4} C\right) .
$$

Let $\Delta=\{1, i\}$ and $S=\{(1,0),(1,1),(1,2),(2,0)\}$. Then

$$
f_{\Delta, S}(x)=\left(x-\overline{(4 i-4)} i(4 i-4)^{-1}\right)(x-1)^{3}=(x+1)(x-1)^{3}
$$

$\left(4 i-4\right.$ is the value of polynomial $(x-1)^{3}$ at $\left.i\right)$ is minimal polynomial of degree 4 , so there is unique polynomial of degree $\leq 3$ such that $f(1)=A, f^{\prime}(1)=B, f^{\prime \prime}(1)=C$ and $f(i)=D$. The monic polynomial $L_{0}(x)$ such that $L_{0}^{\prime}(1)=L_{0}^{\prime \prime}(1)=L_{0}(i)=0$ is

$$
L_{0}(x)=x^{3}-3 x^{2}+3 x-4 i+3 .
$$

The monic polynomial $L_{1}(x)$ such that $L_{1}(1)=L_{1}^{\prime \prime}(1)=L_{1}(i)=0$ is

$$
L_{1}(x)=x^{3}-3 x^{2}-x+3 .
$$

The monic polynomial $L_{2}(x)$ such that $L_{2}(1)=L_{2}^{\prime}(1)=L_{2}(i)=0$ is

$$
L_{2}(x)=(x+1)(x-1)^{2}=x^{3}-x^{2}-x+1 .
$$

The monic polynomial $L_{3}(x)$ such that $L_{3}(1)=L_{3}^{\prime}(1)=L_{3}^{\prime \prime}(1)=0$ is

$$
L_{3}(x)=(x-1)^{3}=x^{3}-3 x^{2}+3 x-1,
$$

So, desired interpolation polynomial is

$$
f(x)=A L_{0}(1)^{-1} L_{0}(x)+B L_{1}^{\prime}(1)^{-1} L_{1}(x)+C L_{2}^{\prime \prime}(1)^{-1} L_{2}(x)+D L_{3}^{\prime \prime}(1)^{-1} L_{3}(x) .
$$


In general, we can find the interpolation polynomial for condition from Theorem 4.4

$$
f(x)=\sum a_{\Gamma, S} f_{\Gamma, S},
$$

where $a_{\Gamma, S} \in K$ are coefficients which we will find from condition $f^{\left(r_{s}\right)}\left(x_{i}\right)=y_{i}^{r^{s}}$ and $f_{\Gamma, S}$ is the minimal polynomial of the pair $(\Gamma, S)$ where

$$
\Gamma=\left\{x_{1}, \ldots, x_{i-1}, x_{i+1}, \ldots, x_{k}\right\}
$$

and

$$
S=\left\{\left(r, s_{r}\right) \mid 1 \leq r \leq k, r \neq i, 0 \leq s_{r} \leq n_{k}\right\}
$$

or $\Gamma=\Delta$ and

$$
S=S_{m, \ell}=\left\{\left(r, s_{r}\right) \mid 1 \leq r \leq k, 0 \leq s_{r} \leq n_{k}, 0 \leq s_{\ell} \leq n_{\ell}-m\right\}
$$

where $1 \leq \ell \leq k$ and $1 \leq m \leq n_{\ell}$.

For example: if $\Delta=\left\{x_{1}, x_{2}\right\}$ and $S=\{(1,0),(1,1),(2,0),(2,1)\}$

$$
\begin{aligned}
f(x)=a_{1} f_{\left\{x_{1}\right\},\{(1,0),(1,1)\}}+a_{2} f_{\left\{x_{2}\right\},\{(2,0),(2,1)\}} & \\
& +a_{3} f_{\left\{x_{1}, x_{2}\right\},\{(1,0),(1,1),(2,0)\}}+a_{4} f_{\left\{x_{1}, x_{2}\right\},\{(1,0),(2,0),(2,1)\}} .
\end{aligned}
$$

If $\Delta=\left\{x_{1}, x_{2}\right\}$ and $S=\{(1,0), \ldots,(1, n-1),(2,0)\}$, then

$$
f(x)=A\left(x-x_{1}\right)^{n}+B\left(x-x_{2}\right)+\sum_{i=0}^{n-2} a_{i} f_{\left\{x_{1}, x_{2}\right\}, S_{i}},
$$

where $S_{i}=\{(2,0)\} \cup\{(1, s) \mid 0 \leq s \leq i\}$.

\section{REFERENCES}

1. N. Jacobson: Theory of Rings. Amer. Math. Soc., Providence, 1943.

2. P. M. Conn: Skew Fields. Theory of General Division Rings. Encyclopedia in Math., Vol. 57, Cabridge Univ. Press, Cambridge, 1995.

3. T. Y. LAM: A general theory of Vandermonde matrices. Expositions Math., 4 (1986), $193-215$

4. E. Artin: Geometric Algebra. Interscience Publishers, Inc., 1957.

Faculty of Civil Engineering,

Received October 24, 2006.

University of Belgrade,

Bulevar Kralja Aleksandra 73

11000 Beograd, Serbia

E-mail: eric@grf.bg.ac.yu 